\title{
Uso de espectroscopia de ultravioleta visível (UV-VIS) para a detecção de quercetina em plantas por meio da formação do complexo quercetina-alumínio
}

\author{
UV-Vis spectroscopy to detection of quercetin in plants by formation \\ of quercetin-aluminum complex
}

Recebido em: 23/02/2017

Aceito em: $\quad 25 / 05 / 2017$
Fabiele BERNARDI; Keller Paulo NICOLINI; Jaqueline NICOLINI Instituto Federal do Paraná - IFPR. Av. Bento Munhoz da Rocha, s/n, PRT280, Trevo da Codapar, CEP 85555-000. Palmas, PR, Brasil.

E-mail: jaqueline.nicolini@ifpr.edu.br

\section{ABSTRACT}

Nine plant samples were studied from complexation reaction that occurs between aluminum from aluminum chloride $\left(\mathrm{AlCl}_{3}\right)$ and quercetin. The reaction shows yellow solution, which was spectroscopically evidenced by the bathochromic shift from 360-370 nm to 420-430 nm. Four samples of petals were investigated: white and magenta azalea, burnt yellow lily and dandelion; three leaf samples: aloe, orange and lime; and two peel samples: orange and lime fruits. Among the analyzed samples, only azalea (Rhododendron sp.) white and magenta petals had sufficient quercetin concentration to be detected by UV-Vis spectroscopy and analyzed by quercetin-aluminum complex formation. The interaction between aluminum and quercetin showed a stoichiometry of $2: 1$, with a binding constant of $3.94 \pm 0.34 \mathrm{x}$ $10^{3} \mathrm{~mol} \mathrm{~L}^{-1}$.

Keywords: Natural products; quercetin; antioxidant effect

\section{RESUMO}

Foram estudadas nove amostras de plantas a partir da reação de complexação que ocorre entre alumínio, proveniente do cloreto de alumínio $\left(\mathrm{AlCl}_{3}\right)$ e quercetina, presente no material botânico avaliado. A reação é caracterizada pela formação de uma solução amarela, evidenciada espectroscopicamente pelo deslocamento batocrômico da região de 360-370 nm para 420-430 nm. Foram investigadas quatro amostras de pétalas: azaleia branca e magenta, lírio amarelo queimado e dente-de-leão; três amostras de folhas: babosa, laranja e lima; e duas amostras de cascas de frutos: laranja e lima. Dentre as amostras analisadas, apenas as pétalas de azaleia (Rhododendron sp.) branca e magenta apresentaram concentração de quercetina suficiente para ser detectada e analisada por espectroscopia de UV-Vis pela formação do complexo quercetina-alumínio. A interação entre o alumínio e a quercetina apresenta estequiometria 2:1, com uma constante de ligação de $3,94 \pm 0,34 \times 10^{3} \mathrm{~mol} \mathrm{~L}^{-1}$.

Palavras-chave: Produtos naturais; quercetina; efeito antioxidante. 


\section{Inotama}

\section{INTRODUÇÃO}

A quercetina, ou 3,5,7,3'-4'-pentahidroxiflavona (Figura 1), é um dos flavonois mais comuns (1), encontrado em plantas medicinais, frutos, vegetais e folhas (2). Como a quercetina pertence ao grupo dos flavonoides, apresenta capacidade antioxidante (3). Todavia, estudos recentes têm mostrado que a ação benéfica da quercetina vai além de sua capacidade de capturar radicais livres no metabolismo (4), protegendo o DNA $(5,6)$, apresentando atividade antitumoral e anticarcinogênica $(7,8)$ e anti-inflamatória (9). A quercetina vem sendo introduzida em suplementos vitamínicos e em formulações farmacêuticas (2).

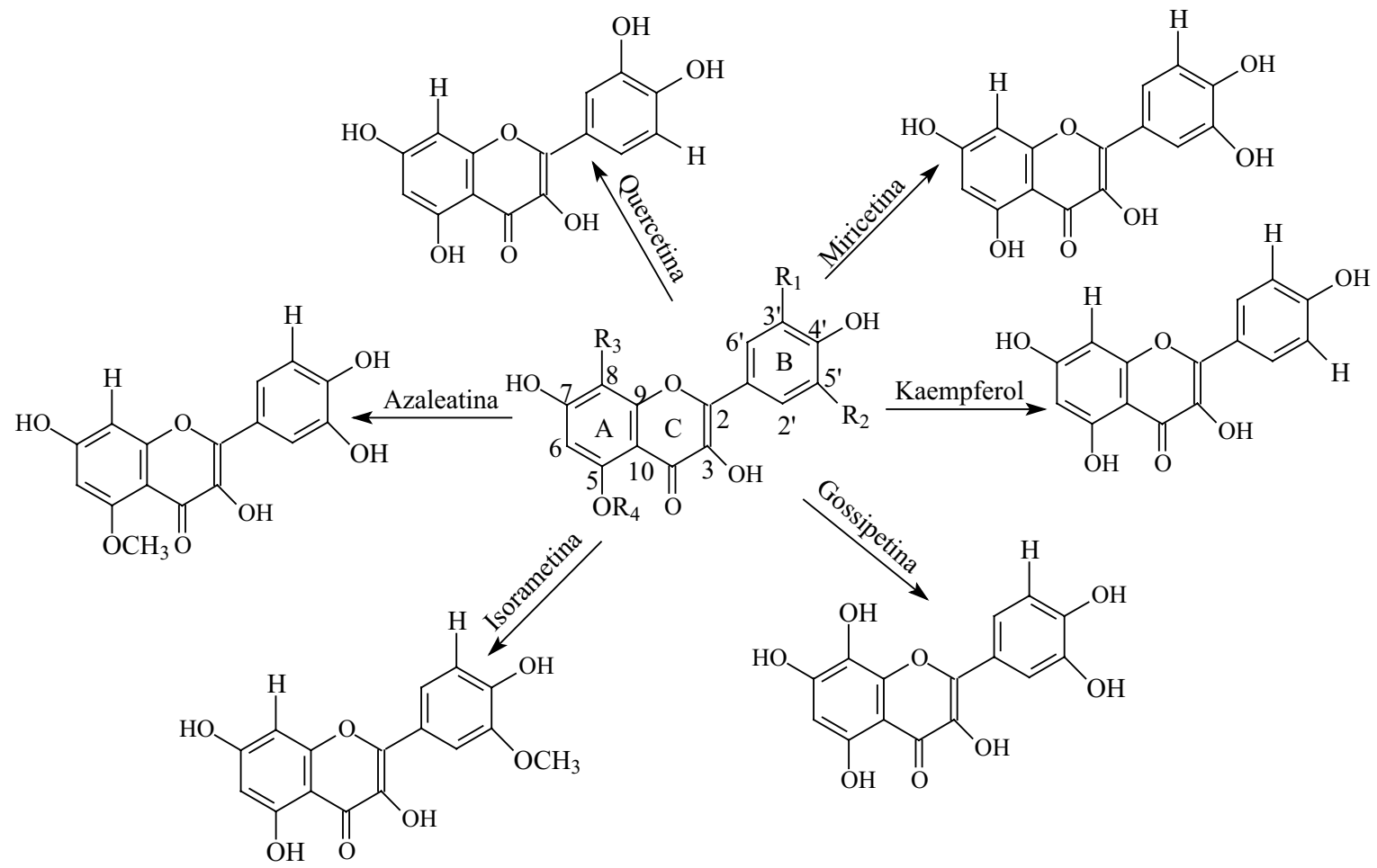

Figura 1. Representação dos flavonois mais comuns

A quercetina é capaz de formar complexos com íons alumínio $\left(\mathrm{Al}^{3+}\right)(10)$ e há indícios de que elevadas concentrações de íons alumínio atuem como neurotoxinas, implicando em distúrbios neurológicos como o Alzheimer (11, 12). Sun e cols. (2016) descreveram a preparação de nanopartículas a base de quercetina, reduzindo os efeitos colaterais do Alzheimer (13). A literatura descreve a detecção de quercetina em várias plantas, utilizando diversos métodos e técnicas de análise (1419), dentre elas a espectroscopia de ultravioleta $(16,20)$.

Esse trabalho tem como objetivo identificar a presença do flavonoide quercetina em plantas, por meio da formação do complexo quercetina:alumínio, utilizando espectroscopia de ultravioleta visível (UV-Vis). As amostras estudadas foram: quatro amostras de pétalas (P): azaleia branca (PAB) (Rhododendron sp.), azaleia magenta (PAM) (Rhododendron sp.), lírio amarelo quei- mado (PLA) (Lilum sp.) e dente-de-leão amarelo (PDL) (Taraxacum sp.); três amostras de folhas (F): babosa (FBB) (Aloe sp.), laranja (FLA) (Citrus sp.) e lima (FLI) (Citrus sp.); e duas amostras de cascas (C) de frutos: laranja (CLA) e lima (CLI). Para compreender a interação que ocorre entre a quercetina e o alumínio, foi determinada a constante de ligação entre a quercetina e íons $\mathrm{Al}^{3+}$ por meio de ajuste não linear.

\section{MATERIAL E MÉTODO}

\footnotetext{
Reagentes, extração da quercetina e curva de calibração.

Foram utilizados os reagentes quercetina (Quercegen, $98,0 \%)$, cloreto de alumínio hexa-hidratado $\left(\mathrm{AlCl}_{3}\right.$ . $6 \mathrm{H}_{2} \mathrm{O}$, Neon, 97,0\%), ácido acético, metanol (Vetec, $\geq$
} 
99,0\%) e ácido clorídrico (Vetec, 37,0\%). Todos os reagentes e solventes foram utilizados sem purificação prévia. Foi pulverizado $0,5 \mathrm{~g}( \pm 0,0001 \mathrm{~g})$ de cada amostra, transferido para um erlenmeyer, com adição de $25 \mathrm{~mL}$ de solução metanólica acidulada $(\mathrm{MeOH}, 1 \% \mathrm{HCl})$, mantida à temperatura de $85 \pm 5{ }^{\circ} \mathrm{C}$ durante 5 horas (21). Os extratos obtidos foram filtrados e transferidos para um balão volumétrico de $25 \mathrm{~mL}$, e o volume foi completado com metanol. Em seguida, uma alíquota de $2 \mathrm{~mL}$ de cada extrato foi transferida para uma cubeta de quartzo de 1 cm de caminho óptico, e diluída para $3 \mathrm{~mL}\left(13,33 \mathrm{~g} \mathrm{~L}^{-1}\right)$ com metanol:ácido acético: $\mathrm{AlCl}_{3}$. Para o preparo dessa solução foi utilizado $0,02 \mathrm{~g}$ de cloreto de alumínio (2000 $\mathrm{mg} \mathrm{L}^{-1} ; 8,28 \times 10^{-6} \mathrm{~mol} \mathrm{~L}^{-1}$ ) dissolvido em $20 \mathrm{~mL}$ de solução de metanol:ácido acético (MeOH:AA 1:19 v/v) (22).

Para a determinação da concentração de quercetina nas amostras, foram construídas duas curvas de calibração utilizando como técnica de análise a espectroscopia de ultravioleta-visível (UV-Vis). Os espectros foram adquiridos em um espectrofotômetro PerkinElmer, Lambda 365 , entre 350 e $550 \mathrm{~nm}$. Foram preparadas duas soluções:

Solução A: Solução de $\mathrm{AlCl}_{3}$, concentração de 8,28 x $10^{-6} \mathrm{~mol} \mathrm{~L}^{-1}\left(2000 \mathrm{mg} \mathrm{L}^{-1}\right)$ dissolvido em MeOH:AA (1:19), contendo quercetina, concentração de 4,94 x 10-4 mol L-1 (100 $\left.\mathrm{mg} \mathrm{L}^{-1}\right)$.

Solução B: Solução de $\mathrm{AlCl}_{3}$ de $8,28 \times 10^{-6} \mathrm{~mol} \mathrm{~L}^{-1}$ (2000 mg L-1) dissolvido em MeOH:AA (1:19), na ausência de quercetina.

As curvas de calibração foram geradas com adições crescentes da Solução A à Solução B. O método avaliou a concentração de quercetina entre as concentrações de $4,94 \times 10^{-7} \mathrm{~mol} \mathrm{~L}^{-1}\left(9,99 \times 10^{-2} \mathrm{mg} \mathrm{L}^{-1}\right)$ e $2,62 \times 10^{-5} \mathrm{~mol}$ $\mathrm{L}^{-1}\left(5,30 \mathrm{mg} \mathrm{L}^{-1}\right)$. As curvas de calibração foram plotadas apenas para as amostras que continham quercetina (PAB e PAM), e apresentaram coeficientes de correlação $\left(\mathrm{R}^{2}\right)$ de $0,99( \pm 0,069)$ para PAB e $0,99( \pm 0,072)$ para PAM. Ambas as curvas de calibração foram plotadas com 31 pontos. Durante a obtenção das curvas de calibração os frascos e a cubeta de quartzo permaneceram hermeticamente fechados com tampas de borracha para evitar a evaporação do solvente, a $25^{\circ} \mathrm{C}$. A curva de calibração para a amostra PAB foi plotada em $422,0 \mathrm{~nm}$ e para a amostra PAM em 430,0 nm.

Determinação da constante de ligação e da estequiometria do complexo formado.

Solução estoque: Para determinar a constante de ligação foram preparados $5 \mathrm{~mL}$ de solução estoque de quercetina $\left(1,0 \times 10^{-2} \mathrm{~mol} \mathrm{~L}^{-1}\right)$ em metanol.
Solução 1: Para o preparo da solução de quercetina $4 \times 10^{-5} \mathrm{~mol} \mathrm{~L}^{-1}$, foi transferida uma alíquota de $40 \mu \mathrm{L}$ da solução estoque para um balão volumétrico de $10 \mathrm{~mL}$, contendo $\mathrm{NaCl}$ para ajustar a força iônica do meio (23) $\left(I=0,1 \mathrm{~mol} \mathrm{~L}^{-1}\right)$, completando o volume com metanol.

Solução 2: A solução de $\mathrm{AlCl}_{3}$ foi preparada na concentração de $1,0 \times 10^{-3} \mathrm{~mol} \mathrm{~L}^{-1} \mathrm{em}$ um balão volumétrico de $5 \mathrm{~mL}$ de $\mathrm{AlCl}_{3}$ a partir da Solução 1.

Em seguida, $2 \mathrm{~mL}$ da Solução 1 foram adicionados em uma cubeta de quartzo hermeticamente fechada com tampa de borracha para evitar a evaporação do solvente, a $25{ }^{\circ} \mathrm{C}$. Com o auxílio de uma micropipeta, a Solução 2 foi adicionada gradativamente à cubeta contendo a Solução 1, para obtenção dos espectros. Os dados espectrais foram coletados e analisados de acordo com as Equações 1 e 2, descritas por Castro e Blanco (2004) (23), que se referem à estequiometria 2:1, sendo:

\section{Equação 1.}

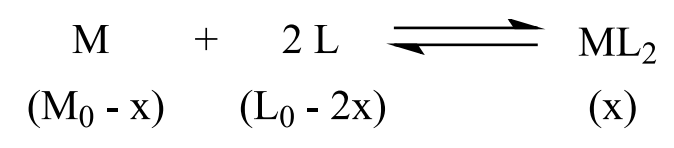

Onde $M$ é a concentração do $\mathrm{AlCl}_{3} ; L$ a concentração de quercetina e $M L_{2}$ a concentração do complexo formado. $M_{0}$ representa a concentração molar de $\mathrm{AlCl}_{3}$ no início do experimento, $L_{0}$ concentração molar de quercetina no início do experimento e $x$ representa a concentração molar do complexo quercetina:alumínio formado.

A constante de ligação $(k)$ foi calculada a partir da Equação 2.

Equação 2.

$$
\frac{A_{e}}{l . M_{0}}=\varepsilon_{C}+\frac{2\left(\varepsilon_{L}-\varepsilon_{C}\right)}{(4 k)^{1 / 3}} \cdot \frac{1}{\left(M_{0}\right)^{2 / 3}}
$$

Onde: $\varepsilon_{L}$ é a absortividade molar do ligante; $\varepsilon_{C}$ a absortividade molar do metal; $l$ a distância do caminho óptico $(1 \mathrm{~cm})$; e $A_{e}$ a absorbância coletada no comprimento de onda máximo. A constante de ligação foi calculada a partir do ajuste não linear dos dados, tendo $A e$ no eixo y versus $M_{0}$ no eixo x.

Para a determinação da estequiometria do complexo formado, foi utilizado o método de Job (método das variações contínuas). Para isso, foram preparadas solu- 
ções equimolares de $\mathrm{AlCl}_{3}$ e quercetina em metanol (4 x $\left.10^{-5} \mathrm{~mol} \mathrm{~L}^{-1}\right)$, contendo $\mathrm{NaCl}\left(I=0,1 \mathrm{~mol} \mathrm{~L}^{-1}\right)$ para manter constante a força iônica do meio. Em seguida, a partir dos comprimentos de onda máximos $\left(\lambda_{\max }\right)$ em 429,3 nm foram plotadas as intensidades de absorção máximas de cada mistura versus as frações molares.

\section{RESULTADOS E DISCUSSÃO}

A adição da solução $\mathrm{MeOH}: \mathrm{AA}: \mathrm{AlCl}_{3}$ confirma a presença de quercetina pelo deslocamento da banda de absorção principal da quercetina, entre $360-370 \mathrm{~nm}$ para maiores comprimentos de onda, entre 420-430 nm (deslocamento batocrômico). Os testes foram realizados para as amostras: PAB, PAM, PLA, PDL, FBB, FLA, FLI, CLA e CLI e o batocromismo foi observado apenas para as amostras PAB e PAM, indicando a presença de quercetina quantificável por este método apenas para estas duas amostras. Os deslocamentos batocrômicos foram de $57 \mathrm{~nm}$ para PAB (de 365,0 nm para $422,0 \mathrm{~nm}$ ) e de $61 \mathrm{~nm}$ para PAM (de $369,0 \mathrm{~nm}$ para $430,0 \mathrm{~nm}$ ). $\mathrm{O}$ deslocamento batocrômico ocorre devido ao cátion alumínio formar complexos estáveis com flavonoides em methanol, provocando alteração na região da banda absorção do anel B da quercetina $(24,25)$. As concentrações de quercetina, para cada grama de pétalas, foram de $0,163 \mathrm{mg} \mathrm{g}^{-1}\left(2,20 \mathrm{mg} \mathrm{L}^{-1} ; 1,09 \times 10^{-5} \mathrm{~mol} \mathrm{~L}^{-1}\right)$ para $\mathrm{PAB}$ e $1,95 \mathrm{mg} \mathrm{g}^{-1}\left(5,20 \mathrm{mg} \mathrm{L}^{-1} ; 2,57 \times 10^{-5} \mathrm{~mol}\right.$ $\left.\mathrm{L}^{-1}\right)$ para PAM. Cao e cols. (18) determinaram a concentração de $5,13 \mathrm{mg} \mathrm{L}^{-1}$ de quercetina em Rhododendron dauricum L. e Liu e cols. (19) identificaram a presença de oito diferentes tipos de quercetina glicosilada em dez espécies de Rhododendron contendo concentrações de quercetina por grama de amostra entre $3,8 \mathrm{mg} \mathrm{g}^{-1}$ e $844 \mathrm{mg} \mathrm{g}^{-1}$.

Esse comportamento pode ser observado devido à formação de um complexo entre a quercetina e os íons $\mathrm{Al}^{3+}$. Uma investigação acerca da estequiometria entre íons $\mathrm{Al}^{3+}$ e quercetina foi realizada, baseada no Método de Job (método das variações contínuas).

$\mathrm{O}$ experimento foi realizado plotando o espectro de UV-Vis de quercetina em metanol na presença e na ausência de íons $\mathrm{Al}^{3+}$, utilizando $\mathrm{NaCl}$ para manter a força iônica do meio constante $\left(I=0,1 \mathrm{~mol} \mathrm{~L}^{-1}\right)$. Os dados coletados indicaram que a quercetina exibe uma forte banda máxima de absorção em $371,6 \mathrm{~nm}$. A adição de íons $\mathrm{Al}^{3+}$ à solução provocou o decréscimo da banda de absorção em 371,6 nm, com formação de nova banda de absorção em 429,3 nm (Figura 2). Os dados coletados em 429,3 nm geraram o gráfico de Job que indicou a razão molar de 0,68 , definindo a razão estequiométrica do complexo Que:Al formado de 2:1 (Figura 2, projeção interna), conforme também descrito por Cornard e Merlin (2002) (10).

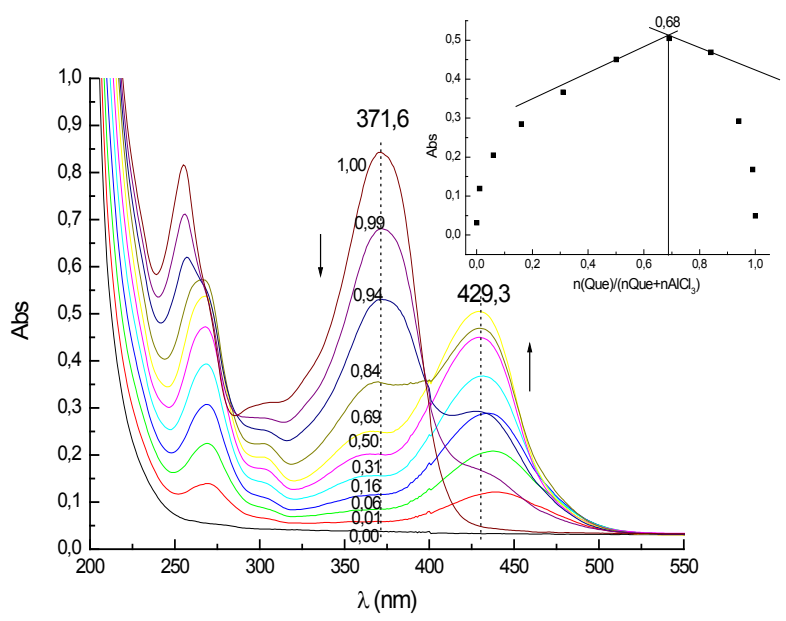

Figura 2. Espectros de UV-Vis da quercetina em metanol na presença e na ausência de íons $\mathrm{Al}^{3+}$. As razões molares entre Que/ $\mathrm{Al}^{3+}$ são indicadas em cada espectro. Projeção interna: Gráfico de Job entre a quercetina (Que) e $\mathrm{Al}^{3+}\left(\mathrm{AlCl}_{3}\right)$ em 429, $3 \mathrm{~nm}$.

Para a determinação da constante de ligação, a concentração de quercetina foi mantida constante, $4 \mathrm{x}$ $10^{-5} \mathrm{~mol} \mathrm{~L}^{-1}$. A concentração máxima de $\mathrm{Al}^{3+}$ adicionada ao final do experimento $4,59 \times 10^{-4} \mathrm{~mol} \mathrm{~L}^{-1}$, o que implica em uma concentração de 11,49 vezes maior de $\mathrm{Al}^{3+}$ em relação à concentração de quercetina (Figura 3). A adição de $\mathrm{Al}^{3+}$ provocou um decréscimo da banda de absorção em 371,6 nm e o surgimento de uma nova banda de absorção em 429,3 nm, com presença de ponto isosbéstico em $392,8 \mathrm{~nm}$, que foi nítido até que a concentração fosse equimolar entre quercetina $\mathrm{e}$ $\mathrm{Al}^{3+}$. Quando a concentração de $\mathrm{Al}^{3+}$ passou a ser 2,27 vezes maior que a concentração de quercetina, começou a surgir um ponto isosbéstico em $434,0 \mathrm{~nm}$ e o aparecimento de uma terceira banda de absorção, em 448,4 nm (Figura 3).

A partir da Equação 2 (23), descrita para estequiometria 2:1, foi possível obter uma constante de ligação de $3,94 \pm 0,34 \times 10^{3} \mathrm{~mol} \mathrm{~L}^{-1}\left(\chi^{2}=2,72 \times 10^{-2}\right)$, a partir de um ajuste não linear (Figura 4). $\mathrm{O}$ ajuste foi realizado para as absorbâncias em $371,6 \mathrm{~nm}\left(A_{e}\right)$, região que indica o desaparecimento da quercetina livre; em 429,3 $\mathrm{nm}\left(A_{e}\right)$ e em $448,4 \mathrm{~nm}\left(A_{e}\right)$, regiões que indicam com- 
plexação entre quercetina e alumínio. A constante que apresentou o melhor ajuste a partir da Equação 2, com menor $\chi^{2}$, foi obtida em $A_{e}=429,3 \mathrm{~nm}$ (Figura 4).

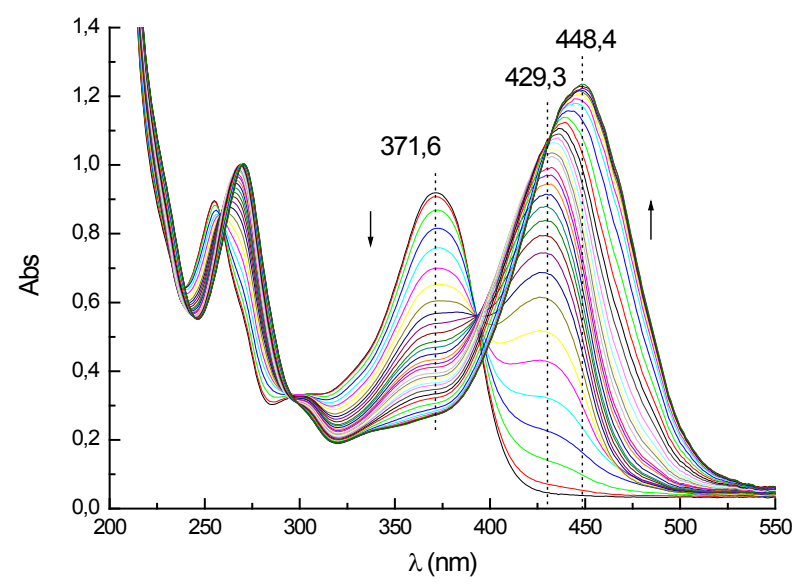

Figura 3. Espectros de UV-Vis de quercetina e cloreto de alumínio (Que:Al) em metanol, a $25{ }^{\circ} \mathrm{C}$, após adições crescentes de $\mathrm{AlCl}_{3^{\prime}}$, sendo a concentração de quercetina constante, $4 \mathrm{x}$ $10^{-5} \mathrm{~mol} \mathrm{~L}^{-1}$.

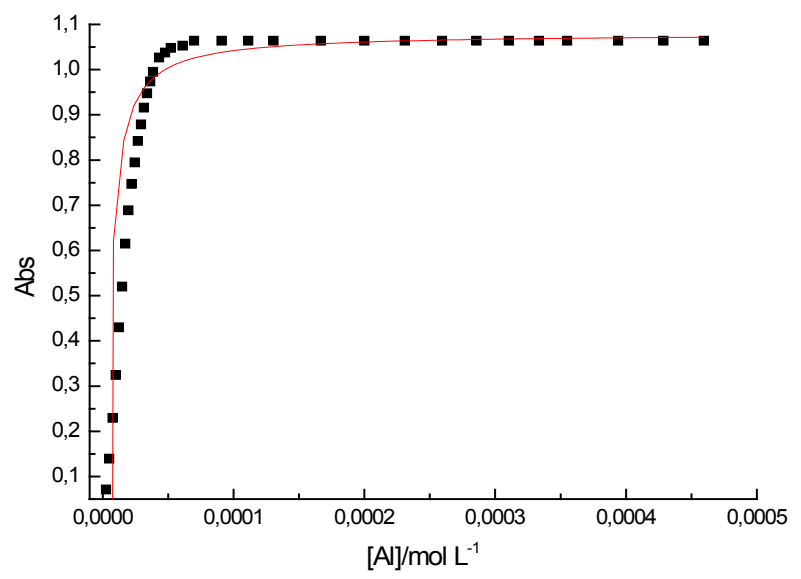

Figura 4. Variação da absorbância em $429,3 \mathrm{~nm}$ pela adição de íons $\mathrm{Al}^{3+}$ à solução contendo quercetina. $\mathrm{A}$ concentração de quercetina se manteve constante $\left(4 \times 10^{-5} \mathrm{~mol} \mathrm{~L}^{-1}\right)$ e a concentração de $\mathrm{Al}^{3+}$ máxima foi de $4,59 \times 10^{-4} \mathrm{~mol} \mathrm{~L}^{-1}$.
Simões (2001) descreveu o potencial efeito inibidor sobre a peroxidação microssomal de lipídeos (atividade antioxidante) que a quercetina e outros flavonoides apresentam (26). No entanto, as plantas do gênero Rhododendron apresentam efeito toxicológico devido à presença de diterpenos derivados do andromedano, de ação cardiotóxica, presentes em todas as partes da planta (26). Isso impede o uso da planta para chás, por exemplo, mas ela mostra ser um removedor natural de íons $\mathrm{Al}^{3+}$ de águas e solos, podendo ser chamado de um "adsorvente verde". Esse estudo indicou que PAB e PAM podem remover $0,18 \mathrm{mg} \mathrm{de} \mathrm{Al}^{3+}$ para cada $\mathrm{mg}$ de quercetina, contida em extratos sólidos. Em amostras líquidas, a PAB pode remover $2,41 \mathrm{mg}$ e a PAM 0,48 $\mathrm{mg}$ de íons $\mathrm{Al}^{3+}$ para cada mg de quercetina.

\section{CONCLUSÃO}

Foi possível identificar a presença de quercetina em azaleia branca e azaleia magenta por meio da formação do complexo quercetina:alumínio, utilizando espectroscopia de ultravioleta visível (UV-Vis). A estequiometria entre alumínio e quercetina foi $2: 1$ com uma constante de ligação de $3,94 \pm 0,34 \times 10^{3} \mathrm{~mol} \mathrm{~L}^{-1}$, a partir de ajuste não linear. Estudos que viabilizem o uso desta técnica no monitoramento de quercetina em plantas por meio do complexo alumínio-quercetina se mostram promissores.

\section{AGRADECIMENTOS}

Os autores agradecem ao Conselho Nacional de Desenvolvimento Científico e Tecnológico (CNPq), ao Instituto Federal do Paraná (IFPR), ao Programa Institucional de Apoio à Pesquisa (PIAP/IFPR - Edital 011/2016) pelo suporte financeiro e ao PIBIC/IFPR pela bolsa de estudos. 


\section{REFERÊNCIAS}

1. Harborne JB. Phytochemical methods: A guide to modern techniques of plant analyses. $3^{\text {th }}$ ed.: Chapman \& Hall: London. 1998, cap. 2.

2. Dmitrienko S, Kudrinskaya V, Apyari V. Methods of extraction, preconcentration, and determination of quercetin. J. Anal. Chem., 2012; 67(4): 299-311. DOI: 10.1134/ S106193481204003X.

3. Hollman PC, Gaag MV, Mengelers MJ, Van Trijp JM, De Vries JH, Katan MB. Absorption and disposition kinetics of the dietary antioxidant quercetin in man. Free Radical Bio. Med., 1996; 21(5): 703-707. DOI: 10.1016/08915849(96)00129-3.

4. Oliveira MR, Nabavi SM, Braidy N, Setzer WN, Ahmed T, Nabavi SF. Quercetin and the mitochondria: A mechanistic view. Biotechnol. Adv., 2016; 34(5): 532-549. DOI: 10.1016/j.biotechadv.2015.12.014.

5. Solimani R. The flavonols quercetin, rutin and morin in DNA solution: UV-vis dichroic (and mid-infrared) analysis explain the possible association between the biopolymer and a nucleophilic vegetable-dye. Bio. Chim. Biophys. Acta, 1997; 1336(2): 281-294. DOI: 10.1016/ S0304-4165(97)00038-X.

6. Das A, Majumder D, Saha C. Correlation of binding efficacies of DNA to flavonoids and their induced cellular damage. J. Photochem. Photobiol., B, 2017; 170(256262. DOI: 10.1016/j.jphotobiol.2017.04.019.

7. Formica J, Regelson W. Review of the biology of quercetin and related bioflavonoids. Food Chem. Toxicol., 1995; 33(12): 1061-1080. DOI: 10.1016/0278-6915(95)000771.

8. Klimaszewska-Wiśniewska A, Hałas-Wiśniewska M, Izdebska M, Gagat M, Grzanka A, Grzanka D. Antiproliferative and antimetastatic action of quercetin on A549 non-small cell lung cancer cells through its effect on the cytoskeleton. Acta Histochem., 2017; 119(2): 99-112. DOI: 10.1016/j.acthis.2016.11.003.

9. Li F, Sun X-Y, Li X-W, Yang T, Qi L-W. Enrichment and separation of quercetin-3-O- $\beta$-d-glucuronide from lotus leaves (Nelumbo nucifera gaertn.) and evaluation of its anti-inflammatory effect. J. of Chromatogr. B, 2017; 1040(186-191. DOI: 0.1016/j.jchromb.2016.12.017.

10. Cornard J, Merlin J. Spectroscopic and structural study of complexes of quercetin with Al (III). J. Inorg. Biochem., 2002; 92(1): 19-27. DOI: 10.1016/S01620134(02)00469-5.

11. Exley C. What is the risk of aluminium as a neurotoxin? What is the risk of aluminium as a neurotoxin?, 2014; 14(6): 589-591.
12. Pogue AI, Lukiw WJ. Aluminum, the genetic apparatus of the human CNS and Alzheimer's disease (AD). Morphol., 2016; 100(329): 56-64. DOI: 10.1016/j.morpho.2016.01.001.

13. Sun D, Li N, Zhang W, Zhao Z, Mou Z, Huang D, Liu J, Wang W. Design of PLGA-functionalized quercetin nanoparticles for potential use in Alzheimer's disease. Colloid Surface B, 2016; 148: 116-129. DOI: 10.1016/j. colsurfb.2016.08.052.

14. Moreira FPM, Coutinho V, Montanher ABP, Caro MSB, Brighente IMC, Pizzolatti MG, Monache FD. Flavonoids and triterpenes from Baccharis pseudotenuifolia: bioactivity on Artemia salina. Quim. Nova, 2003; 26(3): 309311. DOI: $10.1590 / \mathrm{S} 0100-40422003000300004$

15. Molina-Calle M, Priego-Capote F, Luque de Castro MD. Development and application of a quantitative method for determination of flavonoids in orange peel: Influence of sample pretreatment on composition. Talanta, 2015; 144(-): 349-355. DOI: 10.1016/j.talanta.2015.05.054.

16. Nickavar B, Amin G, Mehregan N. Quercetine, a Major Flavonol Aglycon from Tanacetum balsamita L. Iran J. Pharm. Res., 2010; 249-250.

17. Lombard KA, Geoffriau E, Peffley E. Flavonoid quantification in onion by spectrophotometric and high performance liquid chromatography analysis. Hortscience, 2002; 37(4): 682-685.

18. Cao Y, Lou C, Fang Y, Ye J. Determination of active ingredients of Rhododendron dauricum L. by capillary electrophoresis with electrochemical detection. J. Chromatogr. A, 2002; 943(1): 153-157. DOI: 10.1016/S00219673(01)01434-0.

19. Liu L, Zhang L-Y, Wang S-L, Niu X-Y. Analysis of anthocyanins and flavonols in petals of 10 Rhododendron species from the Sygera Mountains in Southeast Tibet. Plant Physiol. Bioch., 2016; 104: 250-256. DOI: 10.1016/j.plaphy.2016.03.036.

20. Silva LAL, Pezzini BR, Soares L. Spectrophotometric determination of the total flavonoid content in Ocimum basilicum L.(Lamiaceae) leaves. Phcog. Mag., 2015; 11(41): 96. DOI: 10.4103/0973-1296.149721.

21. Kurzawa M. Determination of quercetin and rutin in selected herbs and pharmaceutical preparations. Anal. Lett., 2010; 43(6): 993-1002. DOI: 10.1080/00032710903491070. 